\title{
Positron transport and acceleration in beam-driven plasma wakefield accelerators using plasma columns
}

\author{
S. Diederichs, ${ }^{1}$ T. J. Mehrling, ${ }^{2}$ C. Benedetti, ${ }^{2}$ C. B. Schroeder, ${ }^{2}$ A. Knetsch, ${ }^{3}$ \\ E. Esarey, ${ }^{2}$ and J. Osterhoff ${ }^{3}$ \\ ${ }^{1}$ University of Hamburg, Institute of Experimental Physics, D-22761 Hamburg, Germany \\ ${ }^{2}$ Lawrence Berkeley National Laboratory, Berkeley, California 94720, USA \\ ${ }^{3}$ Deutsches Elektronen-Synchrotron DESY, D-22607 Hamburg, Germany
}

(Received 13 March 2019; revised manuscript received 15 May 2019; published 29 August 2019)

\begin{abstract}
A finite radius plasma is proposed to generate wakefields that can focus and accelerate positron beams in a plasma wakefield accelerator. The finite radius plasma reduces the restoring force acting on the plasma electrons forming the plasma wakefield, resulting in an elongation of the on-axis return point of the electrons and, hence, creating a long, high-density electron filament. This results in a region with accelerating and focusing fields for positrons, allowing for the acceleration and quality-preserving transport of high-charge positron beams.
\end{abstract}

DOI: 10.1103/PhysRevAccelBeams.22.081301

\section{INTRODUCTION}

Plasma-based particle accelerators have attracted great interest owing to their high acceleration gradients which could enable a future compact linear electron-positron collider [1]. In a plasma wakefield accelerator (PWFA), an ultrarelativistic, high-density, charged-particle beam propagates through a plasma driving a wake which can sustain large electric fields. In the highly nonlinear blowout [2] or bubble [3] regime all plasma electrons are expelled from the drive beam propagation axis and an ion cavity is formed. This cavity features a region with a transversely linear restoring force for relativistic electrons, together with large accelerating gradients.

While high energy gain and high-efficiency electron acceleration in PWFAs has been demonstrated experimentally $[4,5]$, positron acceleration and transport remains a critical challenge [6]. In the electron-beam driven blowout regime, the focusing region for positrons (located in the narrow cusp where the plasma electrons cross the beam propagation axis behind the blowout) is small, such that stable positron acceleration is highly challenging. A robust concept for positron acceleration and transport is vital for the realization of future PWFA-based colliders.

Previous works have proposed to employ hollow core electron-drive beams [7], positron drive beams [8], or hollow core plasma targets [9] for the generation of wakefields suitable for positron transport and acceleration. However,

Published by the American Physical Society under the terms of the Creative Commons Attribution 4.0 International license. Further distribution of this work must maintain attribution to the author(s) and the published article's title, journal citation, and DOI. these concepts seem impractical and/or entail other challenges. Using hollow core electron drive beams entails the challenge of the generation of these exotic beams and the challenge of preserving their shape. Moreover, the energy conversion efficiency demonstrated in this concept was only at the per-mille-level [7]. The possibility of using positron drive beams has been explored [8]. This method leads to the formation of a considerably long focusing region for positrons, but the nonlinearity of the transverse focusing fields and their variation as the drive beam evolves renders the preservation of the witness beam emittance challenging. Employing hollow core plasma channels allows for the generation of wakefields with substantial longitudinal component and vanishing transverse component [10]. However, owing to the absence of any focusing fields for the drive beam, this scheme suffers from severe beam breakup instability $[10,11]$. Tailored laser profiles have also been proposed to facilitate laser-plasma acceleration of positron beams $[12,13]$.

In this article, we propose a novel method for the generation of wakefields suitable for both the acceleration and transport of positron beams. The method relies on an electron drive beam and a finite-radius plasma column as the target. We investigate the formation of the focusing wake for positron beams by means of particle-in-cell (PIC) simulations, study the emittance preservation analytically, and demonstrate the quality-preserving acceleration and transport of a positron beam. The proposed method addresses a critical challenge for PWFAs and is realizable in existing [14] and soon to be commissioned [15] experimental facilities.

This article is organized as follows. In Sec. II we describe the generation of positron-beam-focusing wakes using plasma columns. The transport and acceleration properties of positron beams in these structures is estimated analytically 
and validated by PIC simulations in Sec. III. Conclusions are presented in Sec. IV.

\section{GENERATION OF POSITRON-BEAM- FOCUSING WAKES USING PLASMA COLUMNS}

The transverse wakefield generated by a dense electron drive beam in a homogeneous plasma target has a linear radial dependence within the ion cavity $[16,17]$, and rapidly decays within the surrounding electron sheath [17]. In contrast, for a plasma with a finite radial extent, $R_{p}$, smaller than the ion cavity, or blowout, radius $R_{b}$ (we assume the drive beam propagates along the column center), the transverse wakefield increases linearly ( $\alpha r$, where $r$ is the distance from the plasma column center) only within the plasma column, and falls off as $\propto r^{-1}$ for $R_{p}<r \leq R_{b}$. For $r>R_{b}$ the wakefield is damped by the electron sheath. The fact that the fields falls off for $r>R_{p}$ implies that the resulting blowout radius is greater than for the homogeneous plasma case, and, most importantly, the altered transverse wakefield structure leads to a modification of plasma electron trajectories such that the longitudinal position behind the drive beam where the electrons cross the $r=0$ axis strongly depends on the particle's impact parameter. This is illustrated in Fig. 1, which shows the plasma electron density and plasma electron trajectories within the $(\zeta, x)$ plane calculated using the quasi-static three-dimensional (3D) PIC code HiPACE [18]. Here $x$ is the transverse distance from the column axis, $\zeta=z-c t$ is the comoving coordinate ( $z$ and $t$ are, respectively, the longitudinal coordinate and the time, and $c$ is the speed of light in vacuum). The colored lines depict trajectories of electrons with varying initial impact parameters, and the plasma electron density is shown in the background (blue color scale). In this example (Figs. 1 and 2), the plasma column

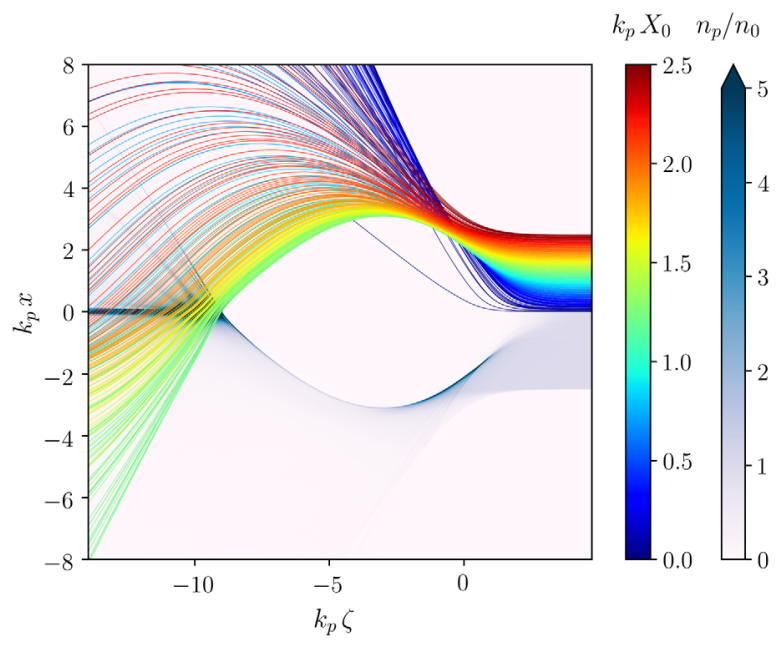

FIG. 1. Plasma electron density, $n_{p} / n_{0}$ (blue color scale), and trajectories of plasma electrons with differing impact parameters, $k_{p} X_{0}$ (colored lines) in a finite radius plasma with $k_{p} R_{p}=2.5$. See text for drive beam parameters. has a radius $k_{p} R_{p}=2.5$, where $k_{p}=\omega_{p} / c$ is the plasma wavenumber, $\omega_{p}=\left(4 \pi n_{0} e^{2} / m\right)^{1 / 2}$ the plasma frequency, $n_{0}$ the plasma electron density within the column, and $e$ and $m$ the electron charge and mass, respectively. The drive beam is Gaussian with dimensions $k_{p} \sigma_{x, y}^{(\mathrm{d})}=0.3, k_{p} \sigma_{\zeta}^{(\mathrm{d})}=$ $\sqrt{2}$, and peak current $\hat{I}_{b} / I_{A}=1$, where $I_{A}=m c^{3} / e \simeq$ $17 \mathrm{kA}$ is the Alfvén current. Instead of the small electron cusp, or density spike, as obtained in a homogeneous plasma, an extended high-density electron filament is generated for $k_{p} \zeta \lesssim-9.0$. This plasma filament induces a long region that is both focusing and accelerating for positron beams, as shown in Fig. 2, depicting the transverse wakefield, $\left(E_{x}-B_{y}\right) / E_{0}$ in the $(\zeta, x)$ plane, together with an on-axis line-out of the longitudinal wakefield, $E_{z} / E_{0}$, where $E_{0}=m c \omega_{p} / e$.

The value of the plasma-column radius strongly affects the wakefield structure. In the limit $R_{p} \gg R_{b}$, the resulting wakefields converge towards the homogeneous plasma case, where the positron focusing and accelerating region becomes very small, existing only within a large electron density spike at the back of the ion cavity. For $R_{p} \lesssim R_{b}$, the opposite occurs, namely the positron-focusing region extends over a progressively longer region while the maximum amplitude of the longitudinal field decreases for smaller $R_{p}$. This is due to the reduction of the restoring forces on the plasma electrons expelled from the drive beam region occurring for smaller column radii.

To investigate this relation more quantitatively, we perform a series of PIC simulations for varying $R_{p}$, using the above mentioned drive beam parameters. For this

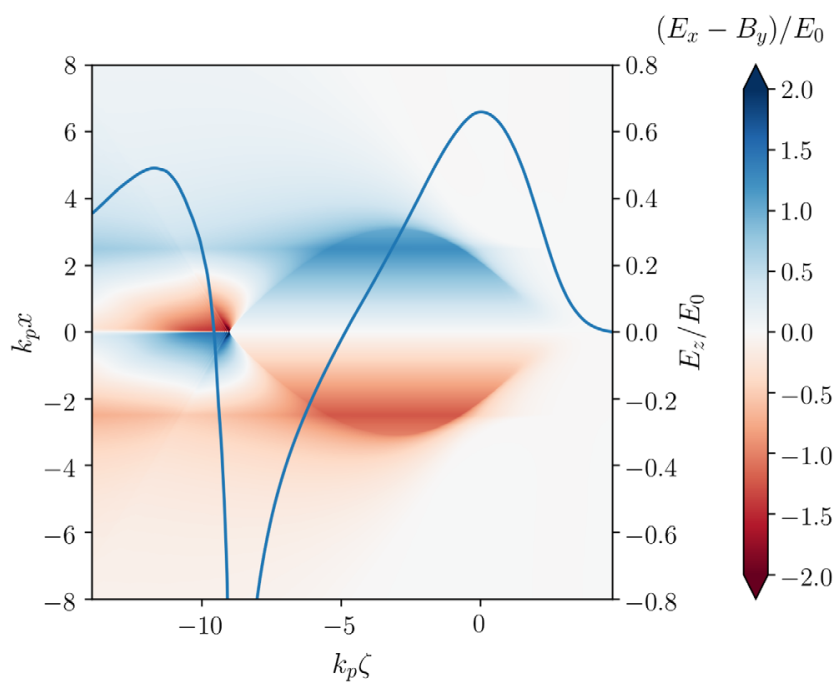

FIG. 2. 2D map of the transverse wakefield $\left(E_{x}-B_{y}\right) / E_{0}$ and on-axis line-out of $E_{z} / E_{0}$ (blue curve) generated by an electron drive beam in a finite plasma column with radius $k_{p} R_{p}=2.5$. Drive beam parameters are the same as in Fig. 1. The finite plasma column leads to the formation of a wide longitudinal region that is both accelerating and focusing for positron beams. 
parameter scan, the quasi-static modality of the cylindrically symmetric PIC code INF\&RNO [19,20] was used, after successfully benchmarking the results against HiPACE. Results are shown in Fig. 3, where the on-axis line-out of the longitudinal field is plotted as a function of the column radius, and where the longitudinal regions that are focusing or defocusing for positrons have been highlighted. For $k_{p} R_{p} \lesssim 2.5$ there is an extended $\zeta$-region allowing for the transport and acceleration of positron beams. The accelerating field amplitude is enhanced for greater plasma radii. However, for $k_{p} R_{p} \gtrsim 2.5$, the positron-focusing region decays to a singular point, rendering stable positron acceleration impossible.

The elongation of the electron trajectories depends on the plasma column radius and on the drive beam parameters. The plasma radius can be optimized for positron acceleration and focusing (i.e., a long focusing $\zeta$-region and high field amplitude) for any given drive beam parameters. In particular, for a Gaussian drive beam with $k_{p} \sigma_{x, y}^{(\mathrm{d})} \ll 1$ and a beam current $1 \leq \hat{I}_{b} / I_{A} \leq 10$, numerical exploration of the parameters space provides the estimate for the optimal plasma radius

$$
k_{p} R_{p} \approx 2 \sqrt[3]{2 \hat{I}_{b} / I_{A}}
$$

This relation ensures that the plasma column radius $R_{p}$ is smaller than the blowout radius $R_{b}$ (compare, e.g., [21,22]) such that the focusing wake region is long and $E_{z}$ in this region is significantly high. For simplicity, we only consider plasma columns with a steplike edge, but it should be noted that this concept is also viable for the case of plasma profiles with a smooth edge.

Such plasma columns can be created either by laserinduced ionization or by beam-field-induced ionization

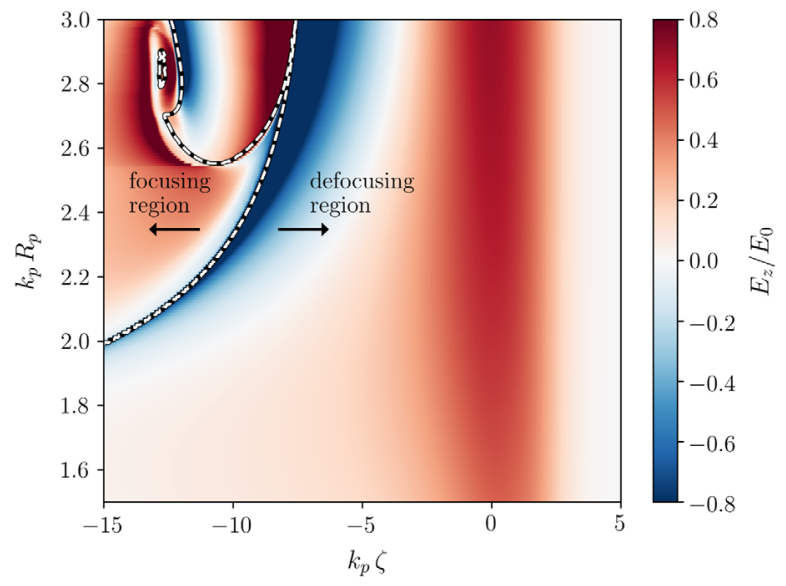

FIG. 3. On-axis longitudinal line-out of the accelerating field, $E_{z} / E_{0}$, plotted as a function of the plasma column radius, $k_{p} R_{p}$. Red regions are accelerating for positrons. The separation between focusing and defocusing regions for positrons is marked with a dashed line. from the drive beam itself. Production of meter-scale plasma columns by laser-induced ionization using an axicon lens [23] has been experimentally demonstrated [24]. The generation of plasma columns with a radial dimension of a few tens of microns and an on-axis density of $\sim 10^{17} \mathrm{~cm}^{-3}$ (parameters near those considered in this work) have also been demonstrated, for the purpose of laser guiding over $\mathrm{cm}$-scale plasmas $[25,26]$. The generation of meter-scale plasma columns, with a few tens of microns radius and an on-axis density of $\sim 10^{17} \mathrm{~cm}^{-3}$, using an axicon lens is possible with current laser technology, requiring on the order of a few $\mathrm{mJ}$ of laser energy per $\mathrm{cm}$ of plasma [26]. On the other hand, creating the plasma column by means of beam-field-induced ionization from the drive beam, instead of relying on an ionizing laser, has the advantage that the column is inherently aligned with the drive beam itself; however, since the ionization rate is strongly coupled to the drive beam parameters and to the gas density, the viable parameter space is limited.

\section{TRANSPORT AND ACCELERATION OF POSITRON BEAMS}

Figure 4 shows a lineout of the transverse wakefield structure (blue curve) at $k_{p} \zeta=-11.6$ for the drive beam parameters considered in Figs. 1 and 2 and a plasma column radius of $k_{p} R_{p}=2.5$. Near the axis, the wakefield has a discontinuous, steplike dependence from the transverse coordinate, while the field strength decays far from the axis. An initially Gaussian witness beam can be quasimatched in such a steplike confining wake so that the emittance growth is minimized during beam transport. The (normalized) rms beam emittance in the $x$-plane is defined as $\epsilon_{x}=\left(\left\langle x^{2}\right\rangle\left\langle u_{x}^{2}\right\rangle-\left\langle x u_{x}\right\rangle^{2}\right)^{1 / 2}$, where $x$ and $u_{x}$ are, respectively, the transverse position and momentum

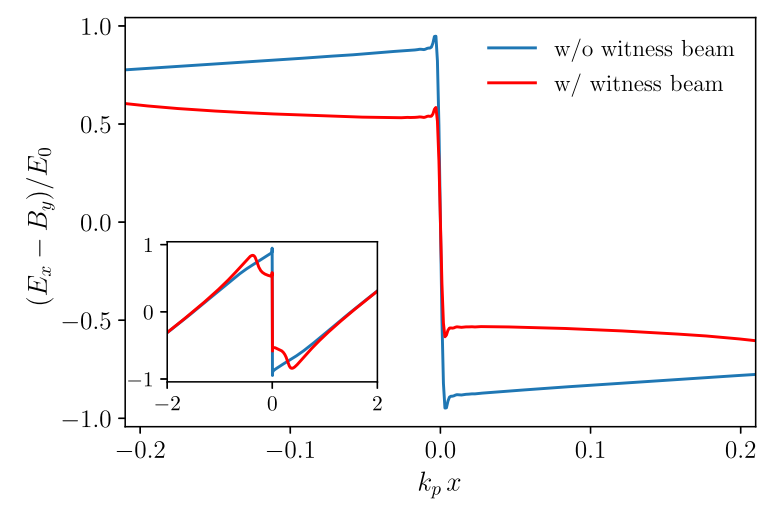

FIG. 4. Transverse wakefield amplitude, $\left(E_{x}-B_{y}\right) / E_{0}$, versus transverse position, $k_{p} x$, for $k_{p} \zeta=-11.6$ (cf., Fig. 2). The blue line depicts the focusing fields without any witness beam, and the red line denotes the focusing fields in the beam-loaded case, where the Gaussian witness beam parameters are given by $\hat{n}_{b} / n_{0}=500, \sigma_{x, y}=0.025 k_{p}^{-1}$, and $\sigma_{z}=0.5 k_{p}^{-1}$. Inset: Transverse wakefields for a wider $x$-range. 
(normalized to $m c$ ) of a beam particle, and where $\langle\cdot\rangle$ refers to the central beam average. A similar definition holds in the $y$-plane. For simplicity, we consider the dynamics of a single beam slice (i.e., we neglect head-tail effects), and we consider a flat beam model (i.e., initially, $\sigma_{x} \gg \sigma_{y}$, and $\sigma_{u_{x}} \gg \sigma_{u_{y}}$, where $\sigma_{u_{x}}$ and $\sigma_{u_{y}}$ are, respectively, the rms transverse momentum spread in the $x$ - and $y$-directions). Extension to a round beam is straightforward following the theory presented in Ref. [27]. Under these hypotheses the motion in the horizontal $x$-plane decouples from the vertical $y$-plane, and is completely determined once the horizontal component of the transverse wake is specified. Following Fig. 4, we model the wake according to a piecewise constant function, namely

$$
\frac{E_{x}-B_{y}}{E_{0}}=-\alpha \operatorname{sgn}(x),
$$

where $\operatorname{sgn}(x)$ is the sign function and $\alpha>0$ is a constant. Assuming the acceleration process is adiabatic, i.e., the single particle action is conserved, the equations of motion (we use $z$, the propagation distance, as the independent variable) describing the orbit of a positron in the $\left(x, u_{x}\right)$ phase-space plane can be derived by the following Hamiltonian,

$$
H\left(x, u_{x}\right)=\frac{u_{x}^{2}}{2 \gamma}+\alpha\left|k_{p} x\right|,
$$

where $\gamma$ is the relativistic Lorentz factor of the beam. For initial conditions $\left(x_{0}, u_{x 0}\right)$, the phase-space orbits for a particle satisfies $u_{x}(x)= \pm\left[2 \gamma \alpha k_{p}\left(x_{\max }-|x|\right)\right]^{1 / 2}$, for $|x| \leq x_{\max }$, where $x_{\max }=H\left(x_{0}, u_{x 0}\right) / \alpha$. From this we can compute the time-average over a closed particle orbit (i.e., the equilibrium value at saturation) of any phasespace-defined dynamic variable. In particular, for the quantities $x^{2}$ and $u_{x}^{2}$, we have $\overline{x^{2}}\left(x_{0}, u_{x 0}\right)=8 x_{\max }^{2} / 15$, and $\overline{u_{x}^{2}}\left(x_{0}, u_{x 0}\right)=2 \gamma \alpha k_{p} x_{\max } / 3$. Finally, by performing an averaging over the initial Gaussian phase-space distribution, we obtain the following expressions for the secondorder phase-space moments at saturation,

$$
\begin{gathered}
\frac{\left\langle x^{2}\right\rangle}{\sigma_{x}^{2}}=\frac{8}{15}\left[1+\sqrt{\frac{2}{\pi}} \eta+\frac{3}{4} \eta^{2}\right], \\
\frac{\left\langle u_{x}^{2}\right\rangle}{\sigma_{u_{x}}^{2}}=\frac{1}{3}\left[1+2 \sqrt{\frac{2}{\pi}} \eta^{-1}\right],
\end{gathered}
$$

where $\eta=\sigma_{u_{x}}^{2} /\left(\gamma \alpha k_{p} \sigma_{x}\right)$. The relative increase of the rms emittance at saturation with respect to the initial emittance is then given by

$$
\begin{aligned}
F(\eta) & \equiv \frac{\left(\left\langle x^{2}\right\rangle\left\langle u_{x}^{2}\right\rangle\right)^{1 / 2}}{\sigma_{x} \sigma_{u_{x}}} \\
& =\left[\frac{8}{45}\left(1+\frac{4}{\pi}+2 \sqrt{\frac{2}{\pi}} \eta^{-1}+\frac{5}{2} \sqrt{\frac{2}{\pi}} \eta+\frac{3}{4} \eta^{2}\right)\right]^{1 / 2} .
\end{aligned}
$$

We see that $F(\eta)>1$ for any value of $\eta$, and so exact rms matching of a beam with initially a transverse Gaussian distribution is not possible. However, $F(\eta)$ has a minimum for $\eta^{*} \simeq 0.72$, where $F\left(\eta^{*}\right) \simeq 1.06$. This means that if, initially, $\sigma_{u_{x}}^{2} \simeq 0.72 \gamma \alpha k_{p} \sigma_{x}$, or, equivalently $\sigma_{x}^{3} \simeq 1.39 \epsilon_{x}^{2} /$ $\left(k_{p} \alpha \gamma\right)$, then the beam is quasi-matched and the rms emittance growth at saturation is the smallest possible $(\simeq 6 \%)$. We verified numerically that a similar matching condition holds in the case of a round beam. In the round beam case, the best matching condition is $\sigma_{u_{x}}^{2} \simeq 0.58 \gamma \alpha k_{p} \sigma_{x}$, or, equivalently,

$$
\sigma_{x}^{3} \simeq 1.72 \frac{\epsilon_{x}^{2}}{k_{p} \alpha \gamma}
$$

and the corresponding emittance growth is $\simeq 2 \%$.

We investigate by means of 3D PIC simulations with HiPACE the quality-preserving acceleration of a positron beam in the wake generated with the method presented in this article. The parameters are as follows. The plasma density is $n_{0}=5 \times 10^{17} \mathrm{~cm}^{-3}$, and the radius of the plasma column is $R_{p}=2.5 k_{p}^{-1} \approx 20 \mu \mathrm{m}$. The drive beam parameters are identical to those used for Figs. 1 and 2. The drive beam is nonevolving in this simulation. A Gaussian positron witness beam copropagates behind the drive beam with a distance between the center of the drive-beam $(\zeta=0)$ and the center of the witness-beam of $11.6 k_{p}^{-1} \approx 88 \mu \mathrm{m}$. The positron beam has an initial rms emittance of $\epsilon_{x}=0.1 k_{p}^{-1} \approx$ $0.75 \mu \mathrm{m}$, an energy of $1 \mathrm{GeV}$, a peak current of $\hat{I}=2.67 \mathrm{kA}$, and an rms bunch length of $\sigma_{z}=0.5 k_{p}^{-1}=3.75 \mu \mathrm{m}$. The transverse wakefield structure within the center of the positron beam has $\alpha \simeq 0.55$. Equation (6) yields $\sigma_{x, y}=$ $0.025 k_{p}^{-1} \approx 0.188 \mu \mathrm{m}$ for the matched $\mathrm{rms}$ beam size (yielding a peak bunch density of $\hat{n}_{b} / n_{0}=500$ ). In the simulation the plasma is sampled with 16 particles per cell, while the drive and witness beams are sampled globally with $10^{6}$ and $3 \times 10^{6}$ constant-weight numerical particles, respectively. The dimensions of the computational domain are $32 \times 32 \times 18.8 k_{p}^{-3}$ and the resolution is $0.0625 \times$ $0.0625 \times 0.0188 k_{p}^{-3}$. In order to resolve the small-scale plasma features present near the axis we use a high-resolution sub-grid embedded within the main computational grid [28]. The sub-grid has the same longitudinal dimension as the main-grid, transverse dimensions of $16 \times 16 k_{p}^{-2}$, and its resolution is $0.0156 \times 0.0156 \times 0.00188 k_{p}^{-3}$.

As discussed above, the Gaussian beam slices cannot be perfectly matched in the wakefield (Fig. 4) and, hence, are 


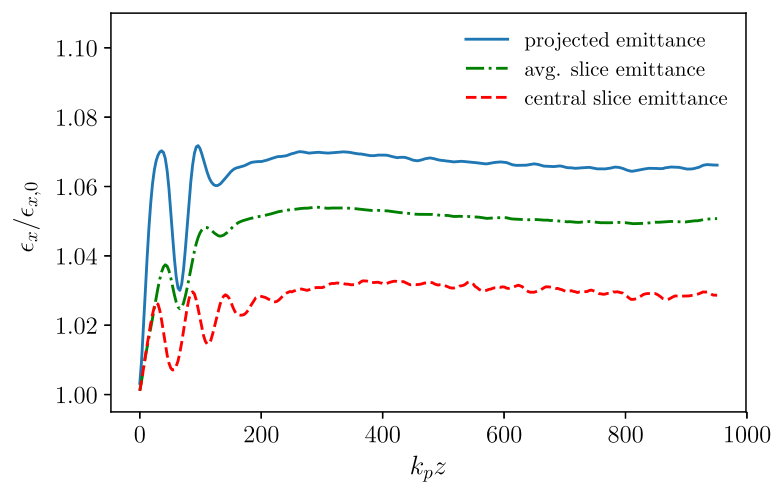

FIG. 5. Emittance evolution of the positron witness beam. Shown are the projected emittance (blue curve), the beamaveraged slice emittance (green curve) and the slice emittance of the central slice (red curve), respectively.

subject to some degree of emittance growth. This can be seen in Fig. 5 (red curve), which shows the evolution of the emittance of the quasimatched central slice in the 3D PIC simulation. The growth saturates at 3\%, in good agreement with the theoretical estimate. For a finite-length beam, each slice is subject to a wakefield with a different strength (i.e., a different $\alpha$ value), such that some parts of the beam do not initially fulfill the matching-condition Eq. (6) and exhibit an emittance growth greater than the one of the central slice. This is shown in Fig. 5 (green curve), indicating that the average slice emittance of the beam grows by $\sim 5 \%$ at saturation. In addition, the variation of $\alpha$ along the beam leads to a head-to-tail betatron decoherence, such that the projected emittance grows by $\sim 7 \%$, as shown in Fig. 5 (blue curve). We note that the emittance growth can be significantly reduced by using a slice-by-slice matched beam (i.e., longitudinally tailoring the beam size, but still retaining a Gaussian distribution for each slice). The emittance growth can be completely suppressed if, for each slice, the transverse phase-space distribution is chosen to be a Vlasov equilibrium for the steplike wakefield instead of a Gaussian distribution, as discussed in Ref. [27].

The longitudinal wakefields generated by this method (cf., Fig. 2) are well-suited for positron beam acceleration. The transformer ratio $R$, defined by the ratio of the peak of the accelerating field of the witness beam and the decelerating field of the drive beam is $R=E_{z \text {,acc }} / E_{z \text {,dec }} \approx 0.7$. The accelerating gradient at the location of the positron beam is $E_{z} / E_{0} \simeq 0.46$ corresponding to $\sim 30 \mathrm{GeV} / \mathrm{m}$. The charge of the drive and witness beams for the chosen plasma density corresponds to $Q_{d} \approx 1.5 \mathrm{nC}$ and $Q_{w} \approx 84 \mathrm{pC}$.

\section{CONCLUSION}

In this work, we demonstrate the transport and acceleration of positron beams by using finite radius plasma targets to control the beam-driven plasma wakefields. We consider an electron drive beam and a positron witness beam, both with Gaussian transverse profiles, propagating in a finite radius plasma, and demonstrate emittance preservation at the percent-level in a strongly loaded wakefield. Further optimization is possible, e.g., the emittance growth can be reduced by slice-by-slice matching of the witness beam to the focusing field [27], and, by proper shaping of drive and witness beams, the transformer ratio and thus efficiency can be increased [29,30]. The initial plasma profile may also be optimized. For example, an exponential decaying plasma for $r>R_{p}$ may allow for an enhanced accelerating field. The optimization regarding these parameters as well as the inclusion of the drive beam evolution will be a subject of future studies. The experimentally feasible approach we have proposed for quality-preserving transport and acceleration of highcharge positron beams in a PWFA solves an outstanding challenge and represents an important step toward future compact plasma-based positron accelerators.

\section{ACKNOWLEDGMENTS}

We gratefully acknowledge Manuel Kirchen, Sören Jalas, and Rémi Lehe for validating our results. We acknowledge the support of the Director, Office of Science, Office of High Energy Physics, of the U.S. Department of Energy, and its Science User Facility National Energy Research Scientific Computing Center (NERSC) under Contract No. DE-AC0205CH11231. We acknowledge the Gauss Centre for Supercomputing e.V. (www.gauss-centre.eu) for funding this project by providing computing time through the John von Neumann Institute for Computing (NIC) on the GCS Supercomputer JUWELS at Jülich Supercomputing Centre (JSC). We acknowledge the Funding by the Helmholtz Matter and Technologies Accelerator Research and Development Program.

[1] C. B. Schroeder, E. Esarey, C. G. R. Geddes, C. Benedetti, and W. P. Leemans, Physics considerations for laserplasma linear colliders, Phys. Rev. Accel. Beams 13, 101301 (2010).

[2] J. B. Rosenzweig, B. Breizman, T. Katsouleas, and J. J. Su, Acceleration and focusing of electrons in two-dimensional nonlinear plasma wake fields, Phys. Rev. A 44, R6189 (1991).

[3] A. Pukhov and J. Meyer-ter Vehn, Laser wake field acceleration: the highly non-linear broken-wave regime, Appl. Phys. B 74, 355 (2002).

[4] I. Blumenfeld, C. E. Clayton, F.-J. Decker, M. J. Hogan, C. Huang, R. Ischebeck, R. Iverson, C. Joshi, T. Katsouleas, N. Kirby, W. Lu, K. A. Marsh, W. B. Mori, P. Muggli, E. Oz, R. H. Siemann, D. Walz, and M. Zhou, Energy doubling of $42 \mathrm{GeV}$ electrons in a metre-scale plasma wakefield accelerator, Nature (London) 445, 741 (2007).

[5] M. Litos, E. Adli, W. An, C. I. Clarke, C. E. Clayton, S. Corde, J. P. Delahaye, R. J. England, A. S. Fisher, J. Frederico, S. Gessner, S. Z. Green, M. J. Hogan, C. Joshi, 
W. Lu, K. A. Marsh, W. B. Mori, P. Muggli, N. VafaeiNajafabadi, D. Walz, G. White, Z. Wu, V. Yakimenko, and G. Yocky, High-efficiency acceleration of an electron beam in a plasma wakefield accelerator, Nature (London) 515, 92 (2014).

[6] H. Fujii, W. An, K. A. Marsh, and C. Joshi, Positron generation and acceleration by injecting two ultra-relativistic intense electron beams upon high-Z target, Bull. Am. Phys. Soc., Div. Plasma Phys. CO7.10 (2018), http:// meetings.aps.org/Meeting/DPP18/Session/CO7.10.

[7] N. Jain, T. M. Antonsen Jr., and J. P. Palastro, Positron Acceleration by Plasma Wakefields Driven by a Hollow Electron Beam, Phys. Rev. Lett. 115, 195001 (2015).

[8] S. Corde et al., Multi-gigaelectronvolt acceleration of positrons in a self-loaded plasma wakefield, Nature (London) 524, 442 (2015).

[9] S. Gessner et al., Demonstration of a positron beam-driven hollow channel plasma wakefield accelerator, Nat. Commun. 7, 11785 (2016).

[10] C. B. Schroeder, D. H. Whittum, and J. S. Wurtele, Multimode Analysis of the Hollow Plasma Channel Wakefield Accelerator, Phys. Rev. Lett. 82, 1177 (1999).

[11] C. A. Lindstrøm et al., Measurement of Transverse Wakefields Induced by a Misaligned Positron Bunch in a Hollow Channel Plasma Accelerator, Phys. Rev. Lett. 120, 124802 (2018).

[12] J. Vieira and J. T. Mendonca, Nonlinear Laser Driven Donut Wakefields for Positron and Electron Acceleration, Phys. Rev. Lett. 112, 215001 (2014).

[13] L.-L. Yu, C. B. Schroeder, F.-Y. Li, C. Benedetti, M. Chen, S.-M. Weng, Z.-M. Sheng, and E. Esarey, Control of focusing fields for positron acceleration in nonlinear plasma wakes using multiple laser modes, Phys. Plasmas 21, 120702 (2014).

[14] A. Aschikhin et al., The FLASH Forward facility at DESY, Nucl. Instrum. Methods Phys. Res., Sect. A 806, 175 (2016).

[15] Technical Design Report for the FACET-II Project at SLAC National Accelerator Laboratory, SLAC Report, 2017.

[16] W. Lu, C. Huang, M. Zhou, W. B. Mori, and T. Katsouleas, Nonlinear Theory for Relativistic Plasma Wakefields in the Blowout Regime, Phys. Rev. Lett. 96, 165002 (2006).

[17] S. A. Yi, V. Khudik, C. Siemon, and G. Shvets, Analytic model of electromagnetic fields around a plasma bubble in the blow-out regime, Phys. Plasmas 20, 013108 (2013).
[18] T. Mehrling, C. Benedetti, C. B. Schroeder, and J. Osterhoff, HiPACE: A quasi-static particle-in-cell code, Plasma Phys. Controlled Fusion 56, 084012 (2014).

[19] C. Benedetti, C. B. Schroeder, E. Esarey, C. G. R. Geddes, and W. P. Leemans, Efficient Modeling of Laser-Plasma Accelerators with INF\&RNO, AIP Conf. Proc. 1299, 250 (2010).

[20] C. Benedetti, C. B. Schroeder, E. Esarey, C. G. R. Geddes, and W.P. Leemans, Efficient modeling of laser-plasma accelerator staging experiments using INF\&RNO, AIP Conf. Proc. 1812, 050005 (2017).

[21] K. V. Lotov, Blowout regimes of plasma wakefield acceleration, Phys. Rev. E 69, 046405 (2004).

[22] A. Martinez de la Ossa, T. J. Mehrling, L. Schaper, M. J. V. Streeter, and J. Osterhoff, Wakefield-induced ionization injection in beam-driven plasma accelerators, Phys. Plasmas 22, 093107 (2015).

[23] J. H. McLeod, Axicons and their uses, J. Opt. Soc. Am. 50, 166 (1960).

[24] S. Green et al., Laser ionized preformed plasma at FACET, Plasma Phys. Controlled Fusion 56, 084011 (2014).

[25] R. J. Shalloo, C. Arran, L. Corner, J. Holloway, J. Jonnerby, R. Walczak, H. M. Milchberg, and S. M. Hooker, Hydrodynamic optical-field-ionized plasma channels, Phys. Rev. E 97, 053203 (2018).

[26] R. J. Shalloo, C. Arran, A. Picksley, A. von Boetticher, L. Corner, J. Holloway, G. Hine, J. Jonnerby, H. M. Milchberg, C. Thornton, R. Walczak, and S. M. Hooker, Low-density hydrodynamic optical-field-ionized plasma channels generated with an axicon lens, Phys. Rev. Accel. Beams 22, 041302 (2019).

[27] C. Benedetti, C. B. Schroeder, E. Esarey, and W. P. Leemans, Emittance preservation in plasma-based accelerators with ion motion, Phys. Rev. Accel. Beams 20, 111301 (2017).

[28] T. J. Mehrling, C. Benedetti, C. B. Schroeder, and E. Esarey, A subgrid algorithm for the efficient modeling of plasmabased accelerators with ion motion using quasi-static particle-in-cell codes, in 2018 IEEE Advanced Accelerator Concepts Workshop (AAC), Breckenridge, CO, USA (2019), https://doi.org/10.1109/AAC.2018.8659404.

[29] P. Chen, J. J. Su, J. M. Dawson, K. L. F. Bane, and P. B. Wilson, Energy Transfer in the Plasma Wake-Field Accelerator, Phys. Rev. Lett. 56, 1252 (1986).

[30] G. Loisch et al., Observation of High Transformer Ratio Plasma Wakefield Acceleration, Phys. Rev. Lett. 121, 064801 (2018). 